\title{
BMJ Open 'Omics' biomarkers associated with chronic low back pain: protocol of a retrospective longitudinal study
}

\begin{abstract}
Massimo Allegri, ${ }^{1,2}$ Manuela De Gregori, ${ }^{3}$ Cristina E Minella, ${ }^{3}$ Catherine Klersy, ${ }^{4}$ Wei Wang, ${ }^{5}$ Moira Sim, ${ }^{5}$ Christian Gieger, ${ }^{6,7}$ Judith Manz, ${ }^{6,7}$ lain K Pemberton, ${ }^{8}$ Jane MacDougall, ${ }^{8}$ Frances MK Williams, ${ }^{9}$ Jan Van Zundert, ${ }^{10}$ Klaas Buyse, ${ }^{10}$ Gordan Lauc, ${ }^{11}$ Ivan Gudelj, ${ }^{11}$ Dragan Primorac, ${ }^{12,13,14,15,16}$ Andrea Skelin, ${ }^{11,12}$ Yurii S Aulchenko, ${ }^{17}$ Lennart C Karssen, ${ }^{17}$ Leonardo Kapural, ${ }^{18}$ Richard Rauck, ${ }^{18}$ Guido Fanelli, ${ }^{1,2}$ on behalf of the PainOMICS Group
\end{abstract}

To cite: Allegri M, De Gregori M, Minella CE, et al. 'Omics' biomarkers associated with chronic low back pain: protocol of a retrospective longitudinal study. BMJ Open 2016;6: e012070. doi:10.1136/ bmjopen-2016-012070

- Prepublication history and additional material is available. To view please visit the journal (http://dx.doi.org/ 10.1136/bmjopen-2016012070).

Received 30 March 2016 Revised 15 July 2016 Accepted 25 August 2016

CrossMark

For numbered affiliations see end of article.

Correspondence to Dr Massimo Allegri; mallegri@parmanesthesia.com

\section{ABSTRACT}

Introduction: Chronic low back pain (CLBP) produces considerable direct costs as well as indirect burdens for society, industry and health systems. CLBP is characterised by heterogeneity, inclusion of several pain syndromes, different underlying molecular pathologies and interaction with psychosocial factors that leads to a range of clinical manifestations. There is still much to understand in the underlying pathological processes and the non-psychosocial factors which account for differences in outcomes. Biomarkers that may be objectively used for diagnosis and personalised, targeted and cost-effective treatment are still lacking. Therefore, any data that may be obtained at the '-omics' level (glycomics, Activomics and genome-wide association studies-GWAS) may be helpful to use as dynamic biomarkers for elucidating CLBP pathogenesis and may ultimately provide prognostic information too. By means of a retrospective, observational, case-cohort, multicentre study, we aim to investigate new promising biomarkers potentially able to solve some of the issues related to CLBP.

Methods and analysis: The study follows a two-phase, 1:2 case-control model. A total of 12000 individuals (4000 cases and 8000 controls) will be enrolled; clinical data will be registered, with particular attention to pain characteristics and outcomes of pain treatments. Blood samples will be collected to perform -omics studies. The primary objective is to recognise genetic variants associated with CLBP; secondary objectives are to study glycomics and Activomics profiles associated with CLBP.

Ethics and dissemination: The study is part of the PainOMICS project funded by European Community in the Seventh Framework Programme. The study has been approved from competent ethical bodies and copies of approvals were provided to the European Commission before starting the study. Results of the study will be reviewed by the Scientific Board and Ethical Committee of the PainOMICS Consortium. The scientific results will be disseminated through peer-reviewed journals.

Trial registration number: NCT02037789; Pre-results.

\section{Strengths and limitations of this study}

- Multiple-centre and multiple-discipline study: The study includes centres in the European Union (EU), USA and Australia, with research teams specialising in following fields: (1) clinical aspects of pain, (2) biology and genetics of pain, (3) generation of '-omics' data and (4) analysis of multiple '-omics' data.

- Hypothesis driven versus hypothesis generating The study aims to profile '-omics' biomarkers (genome-wide association studies (GWAS), glycomics and Activomics) potentially to decipher the pathogenesis of chronic low back pain (CLBP) associated with the different pathophysiological patterns.

- Longitudinal design with a large sample size: (1) discovery phase with 3000 cases and 6000 controls; (2) validation phase with 1000 cases and 2000 controls.

- While the heterogeneity of the study populations is helpful in the discovery phase, this may limit conclusions in the validation phase.

- Functional investigation with animal model has not been included in the current project due to the limitation of the funding.

\section{INTRODUCTION}

Low back pain (LBP) is one of the most common health problems worldwide with an estimated age-standardised point prevalence of $9.4 \% .^{1}$ In 2012, a global review of the prevalence of LBP was published reporting a mean \pm SEM point prevalence of activitylimiting LBP lasting $>1$ day of $11.9 \pm 2.0 \%$, and the 1-month prevalence of $23.2 \pm 2.9 \% .^{2}$ LBP accounts for considerable disability and work absence, and ranks in the Global Burden of Disease 2013 study as a leading contributor to global disability measured in years lost due to disability (YLDs). ${ }^{3}$ LBP is defined as pain 
and discomfort, localised below the costal margin and above the inferior gluteal folds, with or without leg pain.

Prognostic factors for LBP include demographic factors (educational attainment, age and gender), occupational factors (employment), mental health morbidity (anxiety and depression), perception of pain and disability (pain intensity and expectation of persistent pain) and other psychological factors (fear avoidance, catastrophising, illness perceptions) ${ }^{4}$

LBP becomes chronic LBP (CLBP) when symptoms last at least 3 months. Activity-limiting LBP tends to recur and the course of LBP is increasingly viewed as a chronic recurring condition, ${ }^{5}$ which accounts for considerable direct economic costs as well as indirect burdens for society, industry and health systems. ${ }^{6} 7$ The prevalence of chronic CLBP appears to be rising with an increase from $3.9 \%$ in 1992 to $10.2 \%$ in 2006 in the $\mathrm{USA}^{8}$ and from $4.2 \%$ in 2002 to $9.6 \%$ in 2010 in Brazil. ${ }^{9}$

Pain is a subjective sensation, which is influenced by a range of physical and psychosocial factors through poorly understood neural mechanisms. ${ }^{10}$ The advances in medical imaging have improved our ability to identify the anatomic origin of CLBP; however, CLBP is heterogeneous and the anatomic site only tells part of the story. There is considerable variation in prognosis and, at present, CLBP exerts a substantial burden on the individual, the family and workplace.

Evidence-based guidelines recommend the initial exclusion of serious diagnosis before the implementation of clinical management, which promotes continued function and best practice rehabilitation approaches. However, there is much more to understand about the underlying process, how this affects the prognosis and how we can use this to tailor treatment for the individual. In making a diagnosis of LBP, red flag symptoms are used to identify the need for investigation for underlying serious illness. ${ }^{11}$ Yellow flags (psychosocial factors) identify risks of chronicity ${ }^{12}$ and highlight the heterogeneity and complexity of CLBP where the severity, chronicity and prognosis may depend on the anatomical site, the underlying pathological process, comorbidities as well as individual psychosocial factors.

While psychosocial factors clearly influence outcomes in CLBP, genetic and epigenetic factors may account for some variation in response to treatment. Even though persistent CLBP and disc degeneration are known to be heritable $^{13}{ }^{14}$ and the two traits are highly related to one another, with disc degeneration being a major predictor for LBP episodes, ${ }^{14}$ few genetic variants have been identified and confirmed for both traits. ${ }^{15-17}$ Only two genome-wide association studies report on chronic/ persistent widespread pain ${ }^{16}{ }^{17}$ and two genome-wide association studies (GWAS) of intervertebral disc degeneration. ${ }^{18}{ }^{19}$ In keeping with other common complex traits, the individual effects of the identified loci are small and explain only a small fraction of the trait or disease variation. ${ }^{20}$ As such, they do not substantially improve predictions over those based on known factors such as family history. ${ }^{21-23}$ Unfortunately, these data have not yet shed light on the pain pathogenesis mechanisms, and they do not offer prediction of treatment likely suitable in individual patients. Replication of these findings is also needed. Hence, despite promising recent data, new studies are needed to identify objective biomarkers for diagnosis and prediction of treatment's efficacy in patients having CLBP.

Glycomics is an emerging field, recently identified as a priority for the next decade by the US National Academies of Science. ${ }^{24} 25$ Recent studies reported protein glycosylation in large human population samples, with promising glycan profiling for disease diagnosis and stratification for example, autoimmune diseases and haematological cancers, metabolic syndrome, systemic lupus erythematosus and many other diseases. ${ }^{26-33}$ Together with glycomics, Activomics may also provide insight via new biomarkers for LBP as it combines data involved in enzymatic activity of several post-translational modification (PTM) proteins in an integrated model, providing dynamic characterisation of the current state of an organism.

Activomics, is a novel -omic strategy that aims to describe biological systems in terms of differential protein PTM activities. Perturbations in intracellular and/or intercellular cell signalling networks are frequently linked to chronic diseases such as cancer. Enzyme activities in serum are monitored using a proprietary panel of protein and peptide substrates under multiple assay conditions including the judicious use of enzyme cofactors and inhibitors in order to optimise the discrimination between protein modification enzymes with preferences for overlapping primary sequence or structural targets. Principal Activomics substrate panels include proteases (metalloproteinases, serine, cysteine, aspartic proteases) and their protease inhibitors (eg, serpins), caspases, kinases (ser/thr and tyr), phosphatases and (de)acetylases.

Here, we present the study protocol for a retrospective analysis, in a large cohort of patients having CLBP, to determine '-omics' biomarkers (GWAS, glycomics and Activomics) potentially associated with susceptibility to CLBP and with different pathophysiological patterns ${ }^{34} 35$ (see online supplementary file). Glycomic and Activomic approaches aim to reveal alterations in proteome complexity that arise from post-translational modifications that vary in response to changes in the physiological environment, a particularly important avenue to explore in chronic inflammatory diseases. Furthermore, exploring disease-related links between glycomic and Activomic data within the context of a clearly defined genetic and demographic background is a highly original and potentially instructive secondary objective of the current study. Since aforementioned studies connected mostly N-glycans with chronic inflammation and methods for high-throughput glycoprotein analysis are still in development, our glycomic data will be based exclusively on released $\mathrm{N}$-glycans. 
This study will link clinical data to the multiple '-omics' analyses, thereby profiling novel biomarkers, which have strong potential to advance our knowledge of some of the remaining unsolved problems in CLBP.

The present manuscript serves to describe the registered protocol in order to disseminate the rationale, the methods and the main aims of the clinical study.

\section{METHODS}

We present a retrospective observational, multicentre, international clinical study, with a case-control design.

We describe the details of the retrospective cohort protocol clinical study without providing any preliminary results. Patient enrolment is currently active, up to September 2016. The study is part of the PainOMICS project that includes four different trials and was reviewed and funded by European Community in the Seventh Framework Programme (FP7)-THEME (HEALTH.2013.2.2.1-5-Understanding and controlling pain).

The project includes six clinical centres from Italy, Croatia, Belgium, Australia and the USA, and four centres for scientific analyses from Croatia, France, Germany and UK. Statistical expertise is provided by the 'PolyOmica' consulting based in the Netherlands.
The retrospective study was approved by the ethical committees of each separate clinical centre between December 2013 and March 2014.

The study is registered at Clinicaltrials.gov (NCT02037789).

\section{Participant enrolment and data collection}

Cases (patients having CLBP) will be enrolled by each participating centre (figure 1). Every effort will be made to accumulate a well-characterised cohort of patients with persistent CLBP, subgrouped according to the likely anatomical cause of the pain. Patients fulfilling the following conditions will be considered for enrolment: age 18 years and older; chronic pain (pain lasting longer than 12 weeks) between the costal margins and gluteal fold, with or without symptoms in one or both legs, written informed consent signed and Caucasian ancestry.

Controls (patients without CLBP) will be retrieved from two different sources: (1) existing biobanks of healthy participants having collected information about CLBP; (2) participants enrolled in the parallel prospective study on acute LBP (part of PainOMICS projectNCT 02037763), that is, patients who presented with acute LBP and have not become chronic over 6 months
Figure 1 Study flow chart. CRF, Case Report Form; LBP, low back pain.

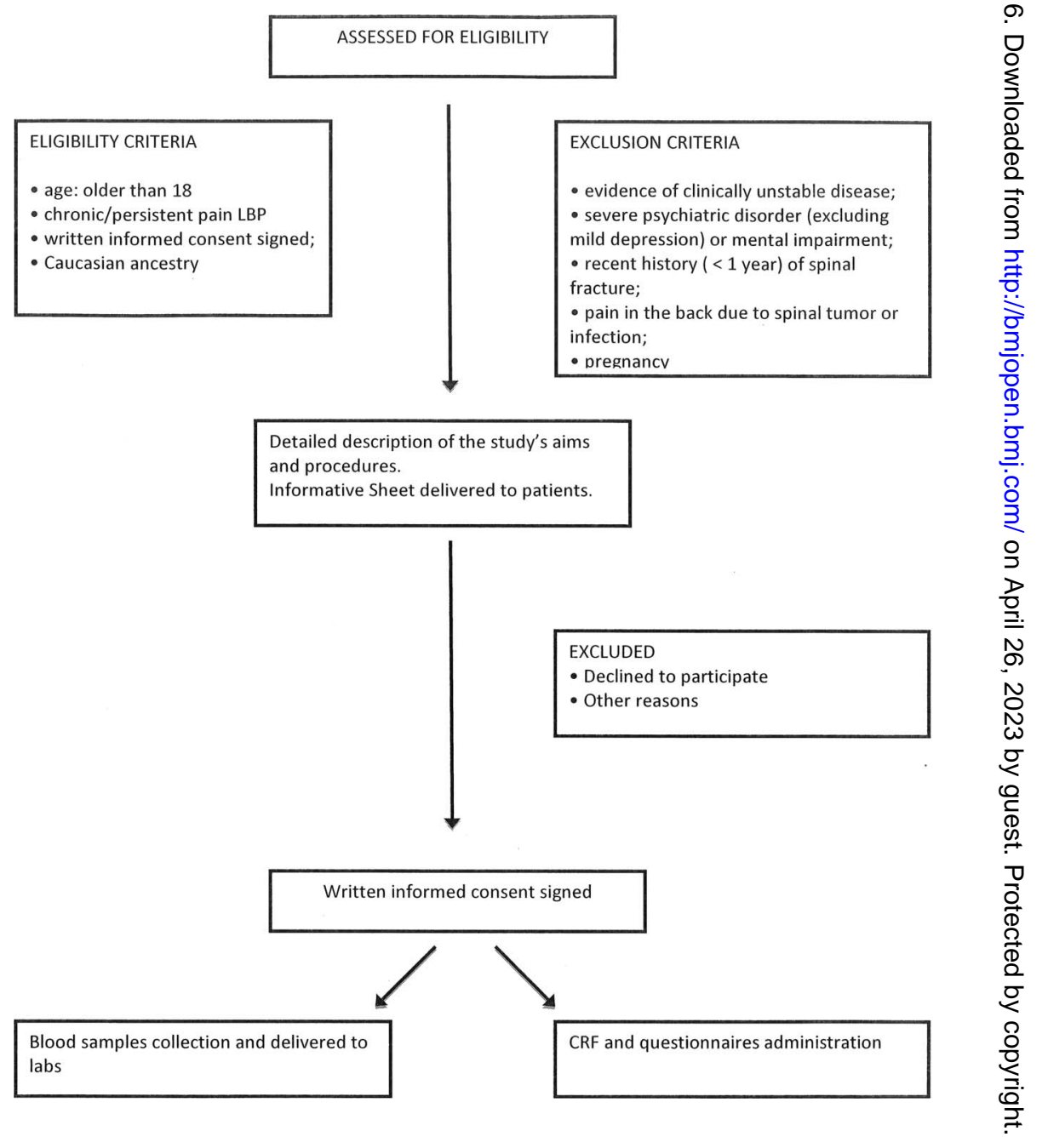


but the pain has resolved. Age (decades) and gender distribution of controls will be matched as closely as possible to that of the cases. Controls are enrolled according to the following inclusion criteria: older than 18 years, no chronic pain (lasting longer than 12 weeks) in the past 12 months, written informed consent obtained and Caucasian ancestry.

Participants with any evidence of clinically unstable disease, severe psychiatric disorder (excluding mild depression) or mental impairment; recent history $(<1$ year) of spinal fracture, back pain due to spinal tumour or infection, pregnancy, will be excluded from the study.

Patients and controls selected for participation will receive a detailed description of the study and will be asked to sign an informed consent prior to entering the study (enrolment visit).

Once enrolled in the trial, patients will be assigned to a unique anonymous code. Data collection includes; demographics (age, gender, race, body mass index (BMI), occupational history), clinical and pharmacological history, pain characteristics (onset, duration, intensity, pain referral pattern, irradiation, sensory abnormalities, precipitating events, history of previous episodes), effectiveness/tolerability of pain treatments received (when applicable). A specific questionnaire (Pain-DETECT (PD)) is applied to evaluate pain type and the pain generator, as well as the possible pathophysiological (nociceptive and/or neuropathic) mechanism sustaining CLBP and functional impairment.

Blood samples for -omics analyses are taken from each enrolled patient at the time of the enrolment consultation, and biological samples are sent to analytical partners of the consortium for specific -omics analysis.

Clinical data are collected in the designated ad-hoc Case Report Form and into a dedicated web database (Research Electronic Data Capture (REDCap)); access to the web database is restricted to the project partners and can be accessed using a dedicated username and a password.

-Omics data are centralised in a specific supervised database.

\section{Samples collection methodology}

All the clinical centres have to guarantee that the biological samples from each patient will be prepared, stored and shipped following the analytical procedures described in three standard operating procedures (SOPs) developed and validated to provide details for conducting the study, phase by phase, with written instructions to achieve uniformity of the procedures used for obtaining patient blood for -omic analysis techniques, storing the samples and shipping the aliquots to the specialised laboratories.

All the patients and controls undergo blood sampling for -omics determinations. The samples will be collected into two tubes containing EDTA for genetic and glycomic analyses and into one serum tube with clot activator plus gel for the Activomics. For genetic analyses, DNA will be isolated from whole blood samples using a commercial DNA extraction kit.

Detailed description of the validation of SOPs is provided in a separate paper under submission.

\section{Analyses for biologic markers}

Genetic analyses

GWAS analyses will be performed on DNA samples isolated from whole blood, using genome-wide Illumina genotyping technology. ${ }^{36}{ }^{37}$ Briefly, $4 \mu \mathrm{L}$ of $60 \mathrm{ng} / \mu \mathrm{L}$ DNA will be amplified at $37^{\circ} \mathrm{C}$ overnight, followed by enzymatic fragmentation, alcohol precipitation and DNA resuspension. Whole genome amplified DNA will be hybridised to the Illumina HumanCore BeadChip, including $>240000$ genome-wide tag single nucleotide polymorphisms (SNPs) and $>20000$ high-value markers (indels and updated exome-focused content). After hybridisation, allelic specificity will be conferred by enzymatic base extension. Products will be subsequently stained, and the intensities of the beads' fluorescence will be detected by the iScan System (Illumina) Genotype calling will be performed with the Illumina GenomeStudio 2011.1 Genotyping Module 1.9.4 software. During quality control genotype data will be filtered by sample-wise and variant-wise call rates and by Hardy-Weinberg Eqilibrium $p$ values. Related individuals, individuals with extreme heterozygosity rates and individuals whose genetics suggest non-Caucasian origins will be discarded. A much denser set of markers will be obtained by genotype imputation using the Haplotype Reference Consortium (HRC) reference panel. The resulting set of markers will contain genotypes measured on the Illumina chip and genotypes imputed based on the very dense HRC reference panel.

For each genetic variant, a standard association model (linear or logistic regression model) will be used to investigate associations between CLBP-related phenotypes and genetic markers. The problem of multiple testing will be addressed by judging the significance of associations using a Bonferroni-corrected, genome-wide significance level corresponding to a nominal significance level of $5 \%$.

\section{Glycomics analyses}

Glycomics analyses will be performed on total serum proteins and on a single serum protein, immunoglobulin $\mathrm{G}$ (IgG). IgG will be isolated from serum samples by affinity chromatography using 96-well monolithic plates with Protein G as previously described (Pucic et al, MCP, 2011). N-glycans will be released from total serum proteins and $\operatorname{IgG}$ by overnight deglycosylation with N-glycosidase F (PNGase F). Released N-glycans will be fluorescently labelled with 2-aminobenzamide (2-AB) fluorescent tag and purified by hydrophilic interaction liquid chromatography (HILIC) solid phase extraction (SPE). Labelled N-glycans will be analysed by hydrophilic interaction chromatography on a Waters Acquity UPLC instrument using Waters BEH Glycan 
chromatography column, $100 \mathrm{mM}$ ammonium formate, $\mathrm{pH} 4.4$, as solvent A and acetonitrile as solvent B. The system will be calibrated using an external standard of hydrolysed and 2-AB-labelled glucose oligomers from which the retention times for the individual glycans will be converted to glucose units. Data processing will be performed with an automatic processing method after which each chromatogram will be manually corrected to maintain the same intervals of integration for all the samples. The chromatograms obtained will all be separated in the same manner into peaks and the amount of glycans in each peak will be expressed as percentage of total integrated area.

\section{Activomics analyses}

Activomics analyses will be performed on retrospective serum samples from anonymous but well-characterised patients. Samples are collected, handled and analysed in a way that minimises freeze-thaw cycles and arrayed with the aid of an automatic liquid handler robot (MultiPROBE II, Perkin Elmer) in 96-well microtitre plates for high throughput screening using microfluidic mobility shift assays. For each enzymatic reaction tested, $2 \mu \mathrm{L}$ of serum from patients and healthy controls will be incubated with the appropriate Activomics substrate under controlled conditions (time, temperature, optimised buffer conditions, etc). In general, the fluorescent peptides to be used as target substrates are synthesised through the addition of an N-terminal carboxyfluorescein (FAM) group via an aminohexanoic acid (Ahx) spacer group, that is, FAM-Ahx-peptide. High throughput screening is performed using a modification of the capillary electrophoretic mobility shift assay on an automated microfluidic platform (EZ Reader, Perkin Elmer, USA). Peptide substrates are generally C-terminally amidated and purified by HPLC to $>90 \%$ purity (GenScript, Hong Kong). All lyophilised peptides are redissolved in sterile, double distilled water at $2 \mathrm{mM}$ concentration and are diluted to $10 \mu \mathrm{M}$ for screening tests. Assays are performed in the appropriate reaction buffer in 384-well format (Corning 3821 BC) using a semiautomated pipettor system for reproducibility (Sorensen Multi (Sorenson Benchtop 96/384 semi-automated pipettor) precision coefficient of variation (CV) $<5 \%$ ). Post-translationally modified products are separated from unreacted substrate by high voltage microfluidic mobility shift assays using voltage and pressure parameters optimised for each substrate. Product conversion is assessed from the respective peak areas obtained from electrophoretic mobility shifts (\% product/substrate+product). The extent of PTM of each substrate is assessed and compared in a univariate analysis for patients with chronic versus those without LBP. The assays will be repeated for a panel of different substrates in order to provide a wide view of disease-related changes to PTM activities for multivariate statistical analysis. Performance characteristics of the panel will be assessed by univariate and multivariate hierarchical clustering and principal component analysis to differentiate activities of disease versus control samples. Sensitivity and specificity will be evaluated in receiver operator characteristic (ROC) analyses to define cut-off values in the design of the optimal predictive biomarker panel.

\section{Primary and secondary objectives}

The primary objective of this retrospective study is to recognise genetic variants associated with persistent CLBP, by comparing patients having CLBP and pain-free patients. We will correlate genetic variants associated with CLBP through a GWAS study, in a wide international population of European ancestry.

Secondary objectives are to recognise glycomic and Activomic data associated with patients having CLBP compared to patients without CLBP.

The participating clinical centres have defined a minimal shared diagnostic data set available in all clinical centres. Each of the participants will stratify patients according to their clinical features, imaging data and results from PD. Considering the patient's response to diagnostic procedures, patients will be subgrouped (taking into account the patient history, clinical examination, radiological results and potentially the response to diagnostic blocks) into six main categories:

1. spinal stenosis,

2. discogenic pain,

3. facet joint pain,

4. sacroiliac joint pain,

5. LBP with radicular pain (not predominant radicular pain),

6. widespread LBP.

\section{Statistics}

Study design

The study will follow a two-phase (discovery and validation), 1:2 case-control model:

- Discovery population: random sample of two-thirds of the entire population of cases.

- Validation population: the remaining one-third. Following the GWAS phase, the genes discovered will be assessed for biological plausibility and entered into the validation phase.

\section{Sample size calculation}

Since, to date, no data on the -omics of CLBP are available in literature, and since the variants associated with most common diseases have modest effects, we considered a number of scenarios, ranging in model assumptions with respect to allele frequency and effect.

With 3000 cases and 6000 controls, we assessed genetic scenarios in which we have $80 \%$ power to detect association at genome-wide significant level. ${ }^{38}$ Consistent with the literature, for high ORs $(=2)$, we will be able to detect variants with low minor allele frequency $(\mathrm{MAF} \geq 1.5 \%)$. With higher allele frequency, we will be able to find smaller effects; for example, for variants with $\mathrm{MAF}=25 \%$, we have power to detect $\mathrm{OR} \geq 1.25$. 
For the replication/validation phase, with 1000 cases and 2000 controls, we have $80 \%$ power to confirm detected variants when using nominal $\mathrm{p}=0.005$ (leading to experiment-wise type I error of 0.05 assuming 10 tests) and $\sim 60 \%$ power in case of more severe multiple testing (100 tests, leading to nominal $\mathrm{p}=0.0005$ ).

\section{Statistical analysis}

All the enrolled patients and controls will be analysed.

\section{Discovery phase}

For analyses investigating highly dimensional -omics space, we will use a range of approaches. The first approach will extend the classical sequential framework. Predictor screening will be performed by logistic or Cox regression models traversing through the -omics space and incorporating few predictors at a time. Statistically significant (at experiment-wise level) predictors will be included in the model, and the next iteration through the -omics space will be performed. A classic example of this approach includes genome-wide association analysis, followed by conditional analyses for identification of secondary signals. To investigate a large numbers of predictors simultaneously, we will use modern regularisation/ shrinkage and machine learning methods allowing analysis of (relatively) large numbers of predictors jointly. While this type of approach does not address the question of statistical testing in the same way as 'classical' approaches do, it is widely used in the context of biomarker discovery, where prediction and not the $p$ values are of primary interest. For all methods aimed towards biomarker discovery, the accuracy of prediction will be accessed by cross-validation, and optimal solutions will be analysed to identify potential biomarkers, which will be selected on their discriminative value in an ROC analysis.

\section{Validation phase}

The discovered genetic variants will be examined bioinformatically for biological plausibility before entering the validation phase. The association of the candidate polymorphisms with the outcome (being a case) will be assessed with logistic regression. The following strategies will be used: single genes assessment/genetic score (sum of candidate genes)/multiple genes. Adjustment for covariates (age, gender, clinical features) will be performed.

Details of the statistical analyses will be provided in the final statistical analysis plan (SAP).

The analysis of the secondary endpoints will follow the same principles reported above.

\section{Ethical issues}

Sample collection and use of clinical data have been started only after the ethical approval of the present study protocol from the competent ethical bodies (ethics committees of the institutions involved in patients' enrolment).
The Scientific Board and Ethical Committee of PainOMICS Consortium will also review the results of the study in order to evaluate any possible societal impact of our findings according to the ethical concerns about genetics/-omics and diagnosis of chronic pain. ${ }^{39}$

\section{Monitoring and quality assessment}

Patients will be withdrawn from the study in case of withdrawal of consent (participants may always without obligation withdraw their informed consent), or any other condition that, on clinical judgement of the investigator, will make unacceptable further study participation for that individual patient.

The Coordinating Investigator (University Hospital of Parma, Italy) will delegate, in each participating centre, a clinical supervisor (to ensure that the study is conducted according to the protocol, good clinical practice and national regulations) and also a data monitor to ensure accuracy, completeness and verification of patients' data. The data monitors, from each participating centre, will make up the data monitoring committee. The External Project Advisory Committee (EPAC) of the PainOMICS FP7 project will perform an overall scientific supervision of the trial and of the emergent data.

The participating members will discuss results and any issues of the study at regular audits during the annual Study in Multidisciplinary Pain Research (SIMPAR) meeting and in any other case that may be deemed necessary.

\section{CONCLUSIONS}

This study is, to the best of our knowledge, the first to investigate genetic and -omic biomarkers in a large population sample of CLPB patients. These biomarkers may be related to pain sensation, as well as to disease pathophysiology and pain generators. The overall objective is to validate associations that may result in a more personalised diagnosis and therapy of a disease with a high health and societal burden such as LBP.

Furthermore, the novel biomarkers emerging from this retrospective study will be validated in a prospective cohort collected within the same PainOMICS project in order to evaluate their ability to predict the possibility of advancement to chronic pain in patients suffering from an acute episode of LBP.

A possible bias could be also related to the fact that in some patients, pain could be still related to acute inflammation even though pain was lasting since $>3$ months However, as we enrol all patients evaluated in chronic pain services who were referred after several pharmacological therapies, we think that this bias will be limited also by the high number of patients enrolled.

The PainOMICS project is expected to significantly expand the level of knowledge on how LBP is generated, propagated and quenched. We will mobilise significant human and material resources in Europe and USA, allowing a comprehensive characterisation of large cohorts of 
patients with CLPB, aiming to identify a number of potential biomarkers related to different aspects of CLBP, as well as potential new targets for therapy.

With this protocol, we would like to investigate better biomarkers related to CLBP. The next mandatory step will be to evaluate if and how these biomarkers could help to predict patients at higher risk of developing chronic pain after an acute episode, and how these biomarkers might also be related to predicting response to pharmacological/surgical treatment. The same research group is already conducting a new prospective study investigating the transition from acute to CLBP and the enrolment will be closed in the spring 2017.

\author{
Author affiliations \\ ${ }^{1}$ Department of surgical science, University of Parma, Parma, Italy \\ ${ }^{2}$ Anesthesia Intensive Care and Pain Therapy service, Azienda Ospedaliera \\ Universitaria Parma, Parma, Italy \\ ${ }^{3}$ Anesthesia, Intensive Care and Pain Therapy, Emergency Department, \\ Fondazione IRCCS Policlinico San Matteo, Pavia, Italy \\ ${ }^{4}$ Research Department, Service of Biometry \& Statistics, Fondazione IRCCS \\ Policlinico San Matteo, Pavia, Italy \\ ${ }^{5}$ School of Medical and Health Sciences, Edith Cowan University, Perth, \\ Western Australia, Australia \\ ${ }^{6}$ Research Unit of Molecular Epidemiology, Helmholtz Zentrum Muenchen, \\ German Research Center for Environmental Health, Neuherberg, Germany \\ ${ }^{7}$ Institute of Epidemiology II, Helmholtz Zentrum Muenchen, German \\ Research Center for Environmental Health, Neuherberg, Germany \\ ${ }^{8}$ Photeomix, IP Research Consulting SAS, Noisy le Grand, France \\ ${ }^{9}$ Department of Twin Research and Genetic Epidemiology, King's College \\ London, London, UK \\ ${ }^{10}$ Department of Anesthesiology, Critical Care and Multidisciplinary Pain \\ Center, Ziekenhuis Oost-Limburg, Genk, Belgium \\ ${ }^{11}$ Genos Glycoscience Research Laboratory, Zagreb, Croatia \\ ${ }^{12}$ St. Catherine Specialty Hospital, Zabok, Croatia \\ ${ }^{13}$ Eberly College of Science, State College, Penn State University, \\ Pennsylvania, USA \\ ${ }^{14}$ Faculty of Medicine, University of Osijek, Osijek, Croatia \\ ${ }^{15}$ University of Split School of Medicine, Split, Croatia \\ ${ }^{16}$ Children's Hospital Srebrnjak, Zagreb, Croatia \\ ${ }^{17}$ PolyOmica, Groningen, The Netherlands \\ ${ }^{18}$ Carolinas Pain Institute, Winston-Salem, North Carolina, USA
}

Acknowledgements Melanie Waldenberger and Concetta Dagostino provided scientific support and are involved in sample management and analysis. Manuela Zunino, Alice Montalti, Dario Bugada, Silvana Montella, Marco Baciarello, Christian Compagnone are involved in data collection and caring for study patients.

Collaborators Melanie Waldenberger (Research Unit of Molecular Epidemiology, Helmholtz Zentrum Muenchen, German Research Center for Environmental Health, Neuherberg, Germany; Institute of Epidemiology II, Helmholtz Zentrum Muenchen, German Research Center for Environmental Health, Neuherberg, Germany), Manuela Zunino (Anesthesia, Intensive Care and Pain Therapy, Emergency Department, Fondazione IRCCS Policlinico San Matteo, Pavia, Italy), Alice Montalti (Anesthesia, Intensive Care and Pain Therapy, Emergency Department, Fondazione IRCCS Policlinico San Matteo, Pavia, Italy), Dario Bugada (Anesthesia Intensive Care and Pain Therapy service, Azienda Ospedaliera Universitaria Parma, Parma, Italy), Concetta Dagostino (Anesthesia Intensive Care and Pain Therapy service, Azienda Ospedaliera Universitaria Parma, Parma, Italy), Silvana Montella (Anesthesia Intensive Care and Pain Therapy service, Azienda Ospedaliera Universitaria Parma, Parma, Italy), Marco Baciarello (Anesthesia Intensive Care and Pain Therapy service, Azienda Ospedaliera Universitaria Parma, Parma, Italy), Christian Compagnone (Anesthesia Intensive Care and Pain Therapy service, Azienda Ospedaliera Universitaria Parma, Parma, Italy).
Contributors MA, CG, IKP, FMKW, JVZ, GL, DP, YSA, LK and GF conceived the study and revised the paper. MA, CEM drafted the paper. MDG, CK, WW, MS, JM, JMD, KB, IG, AS, LCK, RR provided feedback on the manuscript, and all authors reviewed and approved the final version of the paper.

Funding This trial is funded academic/SME research; it is supported by funding from the European Commission in the context of the Seventh Framework Programme of the European Community for Research, Technological Development and Demonstration Activities-(FP7)-THEME (HEALTH.2013.2.2.1-5—Understanding and controlling pain).

Competing interests The study is supported by a grant from the European Commission (602736). MA is a consultant for Grünenthal, Angelini and Mundipharma. He also collaborated for speeches with MSD and CareFusion. GL has multiple patents in the field of glycoscience issued. IKP is the research director of Photeomix, the commercial name of IP Research Consulting SAS, who have ongoing patent applications and trademarks related to Activomics. YSA is a director and co-owner of Maatschap Poly0mica, which provides (consulting) services in the area of (statistical) (gen)omics.

Ethics approval Competent ethical bodies at all the participating clinical centres. Copies of ethical approval were provided to the European Commission before initiating the study. All protocol, copies of informed consent and information sheets approved by the competent ethical bodies, were provided to the European Commission before starting the study.

Provenance and peer review Not commissioned; externally peer reviewed.

Open Access This is an Open Access article distributed in accordance with the Creative Commons Attribution Non Commercial (CC BY-NC 4.0) license, which permits others to distribute, remix, adapt, build upon this work noncommercially, and license their derivative works on different terms, provided the original work is properly cited and the use is non-commercial. See: http:// creativecommons.org/licenses/by-nc/4.0/

\section{REFERENCES}

1. Hoy $D$, March $L$, Brooks $P$, et al. The global burden of low back pain: estimates from the Global Burden of Disease 2010 study. Ann Rheum Dis 2014;73:968-74.

2. Hoy D, Bain C, Williams $G$, et al. A systematic review of the global prevalence of low back pain. Arthritis Rheum 2012;64:2028-37.

3. Collaborators G.B.o.D.S. Global, regional, and national incidence, prevalence, and years lived with disability for 301 acute and chronic diseases and injuries in 188 countries, 1990-2013: a systematic analysis for the Global Burden of Disease Study 2013. Lancet 2015;386:743-800.

4. Campbell P, Foster NE, Thomas E, et al. Prognostic indicators of low back pain in primary care: five-year prospective study. J Pain 2013;14:873-83.

5. Hoy D, Brooks $\mathrm{P}$, Blyth $\mathrm{F}$, et al. The Epidemiology of low back pain. Best Pract Res Clin Rheumatol 2010;24:769-81.

6. Steenstra IA, Verbeek JH, Heymans MW, et al. Prognostic factors for duration of sick leave in patients sick listed with acute low back pain: a systematic review of the literature. Occup Environ Med 2005;62:851-60.

7. Kaplan W, Wirtz VJ, Mantel-Teeuwisse A, et al. Priority medicines for Europe and the World 2013. Upgrade. WHO Library Cataloguingin-Publication 2013;126-8.

8. Freburger JK, Holmes GM, Agans RP, et al. The rising prevalence of chronic low back pain. Arch Intern Med 2009;169:251-8.

9. Meucci RD, Fassa AG, Paniz VM, et al. Increase of chronic low back pain prevalence in a medium-sized city of southern Brazil. BMC Musculoskelet Disord 2013;14:155

10. Koyama T, McHaffie JG, Laurienti PJ, et al. The subjective experience of pain: where expectations become reality. Proc Nat Acad Sci USA 2005;102:12950-5.

11. http://www.sheffieldbackpain.com/professional-resources/learning/ in-detail/red-flags-in-back-pain

12. http://www.sheffieldbackpain.com/professional-resources/learning/ in-detail/yellow-flags-in-back-pain.

13. Battié MC, Videman T, Levalahti $\mathrm{E}$, et al. Heritability of low back pain and the role of disc degeneration. Pain 2007;131:272-80.

14. MacGregor AJ, Andrew T, Sambrook PN, et al. Structural, psychological, and genetic influences on low back and neck pain: a study of adult female twins. Arthritis Rheum 2004;51:160-7. 
15. Eskola PJ, Lemmelä S, Kjaer P, et al. Genetic association studies in lumbar disc degeneration: a systematic review. PLOS ONE 2012;7: e49995.

16. Kim H, Clark D, Dionne RA. Genetic contributions to clinical pain and analgesia: avoiding pitfalls in genetic research. $J$ Pain 2009;10:663-93.

17. Peters MJ, Broer L, Willemen HL, et al. Genome-wide association study meta-analysis of chronic widespread pain: evidence for involvement of the 5 p15.2 region. Ann Rheum Dis 2013;72:427-36.

18. Williams FM, Bansal AT, van Meurs JB, et al. Novel genetic variants associated with lumbar disc degeneration in northern Europeans: a meta-analysis of 4600 subjects. Ann Rheum Dis 2013;72:1141-8.

19. Song $Y Q$, Karasugi T, Cheung KM, et al. Lumbar disc degeneration is linked to a carbohydrate sulfotransferase 3 variant. J Clin Invest 2013;123:4909-17.

20. Visscher PM, Brown MA, McCarthy MI, et al. Five years of GWAS discovery. Am J Hum Genet 2012;90:7-24.

21. Aulchenko YS, Struchalin MV, Belonogova NM, et al. Predicting human height by Victorian and genomic methods. Eur J Hum Genet 2009;17:1070-5.

22. Clayton DG. Prediction and interaction in complex disease genetics: experience in type 1 diabetes. PLoS Genet 2009;5:e1000540.

23. Eichler EE, Flint J, Gibson G, et al. Missing heritability and strategies for finding the underlying causes of complex disease. Nat Rev Genet 2010;11:446-50.

24. National Research Council (US) Committee on Assessing the Importance and Impact of Glycomics and Glycosciences. Transforming glycoscience: a roadmap for the future. Washington DC: National Academies Press (US), 2012. http://www.ncbi.nlm.nih. gov/books/NBK109958/

25. Lauc G, Rudan I, Campbell $\mathrm{H}$, et al. Complex genetic regulation of protein glycosylation. Mol Biosyst 2010;6:329-35.

26. Knezevic A, Polasek O, Gornik O, et al. Variability, heritability and environmental determinants of human plasma $\mathrm{N}$-glycome. $J$ Proteome Res 2009;8:694-701.

27. Lauc G, Huffman JE, Pučic M, et al. Loci associated with $\mathrm{N}$-glycosylation of human immunoglobulin $\mathrm{G}$ show pleiotropy with autoimmune diseases and haematological cancers. PLoS Genet 2013;9:e1003225.

28. Lauc G, Pezer M, Rudan I, et al. Mechanisms of disease: the human N-glycome. Biochim Biophys Acta 2016;1860:1574-82.

29. Lu JP, Knežević A, Wang YX, et al. Screening novel biomarkers for metabolic syndrome by profiling human plasma $\mathrm{N}$-glycans in Chinese Han and Croatian populations. J Proteome Res 2011;10:4959-69.

30. Pabst M, Wu SQ, Grass J, et al. IL-1beta and TNF-alpha alter the glycophenotype of primary human chondrocytes in vitro. Carbohydr Res 2010;345:1389-93.

31. Pucic M, Knezevic A, Vidic J, et al. High throughput isolation and glycosylation analysis of IgG-variability and heritability of the IgG glycome in three isolated human populations. Mol Cell Proteomics 2011;10:M111. 010090.

32. Ruhaak LR, Uh HW, Beekman M, et al. Decreased levels of bisecting GlcNAc glycoforms of IgG are associated with human longevity. PLOS ONE 2010;5:e12566.

33. Vučković F, Krištić J, Gudelj I, et al. Association of systemic lupus erythematosus with decreased immunosuppressive potential of the IgG glycome. Arthritis Rheumatol 2015;67:2978-89.

34. Freidin MB, Lauc G, Allegri M, et al. Using omics in chronic pain conditions to delineate mechanisms and provide new therapeutic strategies. Pain Manag 2016;6:211-15.

35. Freidin MB, Keser T, Gudelj I, et al. The association between low back pain and composition of IgG glycome. Sci Rep 2016;6:26815

36. Scott RA, Lagou V, Welch RP, et al. Large-scale association analyses identify new loci influencing glycemic traits and provide insight into the underlying biological pathways. Nat Genet 2012;44:991-1005.

37. Schaefer AS, Bochenek G, Jochens A, et al. Genetic evidence for PLASMINOGEN as a shared genetic risk factor of coronary artery disease and periodontitis. Circ Cardiovasc Genet 2015;8:159-67.

38. Pe'er I, Yelensky R, Altshuler D, et al. Estimation of the multiple testing burden for genomewide association studies of nearly all common variants. Genet Epidemiol 2008;32:381-5.

39. Compagnone $\mathrm{C}$, Tagliaferri $\mathrm{F}$, Allegri $\mathrm{M}$, et al. Ethical issues in pain and omics research. Some points to start the debate. Croat Med J 2014;55:1-2. 